\title{
Método computadorizado para medida da acuidade visual
}

\author{
A computerized method for visual acuity assessment
}

\author{
Patrícia Katayama Kjaer Arippol ${ }^{1}$ \\ Solange Rios Salomão ${ }^{2}$ \\ Rubens Belfort Jr. ${ }^{3}$
}

Trabalho realizado no Departamento de Oftalmologia da Universidade Federal de São Paulo - UNIFESP.

Ortoptista e Tecnóloga Oftálmica. Mestre em Ciências da Saúde pela Universidade Federal de São Paulo UNIFESP - São Paulo (SP) - Brasil.

Professora Adjunto Livre Docente do Departamento de Oftalmologia da UNIFESP - São Paulo (SP) - Brasil.

Professor Titular do Departamento de Oftalmologia da UNIFESP - São Paulo (SP) - Brasil.

Endereço para correspondência: Patrícia Katayama Kjaer Arippol. 939 Shell Blvd. Apt. 105 - Foster City, CA 94404 - USA

E-mail: patriciarippol@gmail.com

Recebido para publicação em 17.11.2004

Última versão recebida em 17.03.2006

Aprovação em 13.07.2006

Fonte de auxílio à pesquisa: CAPES (para P.K.K.A.)

\section{RESUMO}

Objetivo: Elaborar e validar teste computadorizado para medida da acuidade visual de escolares. Métodos: Foi elaborado teste computadorizado para determinação da acuidade visual utilizando os padrões das tabelas logarítmicas impressas adotadas na clínica oftalmológica. Foram avaliados 90 alunos da primeira série do ensino básico, oito estudantes do curso de Tecnologia Oftálmica da UNIFESP-EPM e 10 pacientes do ambulatório de Estrabismo do Departamento de Oftalmologia da UNIFESPEPM. Todos os sujeitos foram avaliados pelo mesmo examinador e submetidos ao exame de acuidade visual monocular, pela tabela logarítmica de optotipos E impressa e do novo teste computadorizado no mesmo momento. Os participantes forneceram os seus consentimentos após esclarecimento. Resultados: As análises estatísticas revelaram correlação excelente $(r>0,75)$ entre os dois métodos, apesar da leve tendência apresentada pelo teste computadorizado em superestimar a acuidade visual quando comparado com o padrão-ouro. O teste computadorizado apresentou sensibilidade de $100 \%$ e especificidade de $94 \%$. Conclusões: Os resultados obtidos nos permitem dizer que o teste computadorizado pode ser utilizado como novo recurso para triagem da qualidade visual dos escolares, por ser método rápido, de fácil aplicação, barato, automático e atrativo para as crianças. A automatização desvincula o aplicador da interpretação das respostas dadas pelo aluno testado, garante padronização do procedimento, que favorece as análises de acompanhamento e pode ser realizado por diferentes examinadores. Para melhor compreensão da efetividade do teste como instrumento de triagem visual, seria interessante instituí-lo nas escolas do curso básico, após treinamento dos professores para sua aplicação.

Descritores: Acuidade visual; Seleção visual; Validação de programas de computador; Visão; Estudantes

\section{INTRODUÇÃO}

O desenvolvimento da visão ocorre gradualmente a partir do nascimento até aproximadamente os 7 anos de idade, quando atinge o nível de acuidade visual (AV) do adulto $(20 / 20)^{(1)}$. Durante este período o sistema visual está sujeito aos defeitos causados pela estimulação inadequada de um ou ambos os olhos. Um exemplo de alteração da visão é a ambliopia ${ }^{(2-4)}$, defeito do sistema visual central caracterizado pela redução uni ou bilateral da AV corrigida, diminuição da sensibilidade ao contraste e localização espacial, sem causa orgânica detectável através de exame oftalmológico ${ }^{(5-9)}$.

Até a idade escolar, a maior parte das deficiências visuais não são detectadas devido à pouca exigência visual nas atividades do dia-a-dia infantil. Após o ingresso na vida escolar, as atividades curriculares exigem 
performance visual maior e os problemas pré-existentes se manifestam. A detecção precoce das deficiências visuais favorece a recuperação da função normal, evitando que tais alterações se estabeleçam definitivamente como, por exemplo, na ambliopia.

Uma alternativa prática para a detecção precoce de tais deficiências seria a implementação de projetos de triagem visual em pré-escolares com a utilização de métodos rápidos, baratos e eficazes, que pudessem ser aplicados por pessoal não especializado ${ }^{(10-15)}$. Desta maneira estaríamos compartilhando com pessoas de fora da área médica uma tarefa que, se bem executada, alcançaria o mesmo resultado, sem a dependência exclusiva do médico oftalmologista ou de outros profissionais da área da saúde.

Os programas atuais de triagem visual de pré-escolares são aplicados por professores que, após receberem treinamento, determinam a AV dos alunos com a utilização das tabelas impressas com os Optotipos de Snellen ${ }^{(16-19)}$. Algumas dúvidas relacionadas ao método de aplicação e interpretação das respostas dos alunos e quanto ao tipo de tabela utilizada nesses programas, colocam em questão sua eficácia ${ }^{(11)}$.

A medida para solucionar tais dúvidas seria o desenvolvimento de programas computadorizados para medida da acuidade visual, com rígido controle das variáveis que possam interferir na qualidade da medida proposta. O controle do estímulo apresentado permite reduzir a memorização da sequência dos optotipos, realizar mudança instantânea do contraste, variar o tamanho das letras, alternar as apresentações entre linear e angular, inserir barras de interação, desta forma diminuindo a variabilidade das medidas obtidas com as tabelas e projetores geralmente utilizados. A possibilidade de gravação em computador da medida da AV para futuras análises e comparações, ou seja, a automatização do teste de AV, faz com que ele se torne objetivo, com resultados independentes da interação do examinador, além de ser mais atraente e estimulante para as crianças e de reduzir o tempo de exame. O teste automatizado também favorece o transporte e o armazenamento dos dados, que podem ser gravados em disquetes ou enviados através da Internet ${ }^{(12)}$.

O objetivo deste estudo foi a elaboração de um novo programa computadorizado que possibilite a medida da AV com apresentação de estímulos logarítmicos elaborados no formato de optotipos "E”.

\section{MÉTODOS}

Este projeto de pesquisa foi submetido à avaliação do Comitê de Ética em Pesquisa da UNIFESP-EPM, tendo sido aprovado em 09/11/2001.

O programa computadorizado foi idealizado para ser de fácil e correta aplicação por professores de escolas primárias, com o objetivo de realizar uma medida fiel da AV e determinar se ela é normal ou não. Foi desenvolvido em parceria com o Departamento de Informática em Saúde da UNIFESP-EPM, em linguagem "Flash", que permite a apresentação de animações gráficas, com recursos multimídia de fácil manuseio e que pode ser instalado em qualquer computador.

O programa computadorizado apresenta, na página de teste, 11 fileiras de optotipos "E" com 5 letras cada. O tamanho das letras varia de 0,7 $\log$ MAR $(21,816 \mathrm{~mm}$ de altura) a $-0,3$ $\log$ MAR $(2,1816 \mathrm{~mm}$ de altura), respeitando a regressão logarítmica de $0,1 \log$ por linha.

O tamanho das letras foi calculado com base no ângulo visual de 5 minutos de arco, formado no ponto nodal do olho quando visto a 3 metros de distância.

Para a construção da tabela computadorizada, considerouse a resolução mais freqüente dos monitores de computadores e o tamanho em polegadas. Os optotipos são representados por pontos pretos no monitor, conhecidos como pixels (elementos do desenho $)^{(13)}$. Para resolução 640x480 de um monitor de 14 polegadas, cada "pixel" (elemento do desenho) corresponde a aproximadamente $0,42 \mathrm{~mm}$; para um monitor de 15 polegadas de mesma resolução, cada "pixel" corresponde a aproximadamente 0,44 mm. Para resolução 800x600 de um monitor de 14 polegadas, cada "pixel" corresponde a aproximadamente $0,33 \mathrm{~mm}$ e monitor de 15 polegadas a aproximadamente $0,35 \mathrm{~mm}$. O programa fornece a opção de embaralhar os optotipos durante o teste, para evitar memorização da sequiência de posicionamento (Figura 1).

A tabela de optotipos impressa:

A tabela de optotipos "E" é formada por 12 fileiras com 5 letras cada, com tamanho das letras variando em progressão logarítmica de 1,0 logMAR (20/200) a -0,1 logMAR (20/10). O espaçamento entre letras em cada fileira mantém a mesma largura das letras e o espaçamento entre fileiras corresponde ao tamanho das letras da menor fileira. Essa tabela foi adotada como padrão-ouro durante a pesquisa.

Foram avaliados 108 indivíduos voluntários divididos em 3 grupos:

Grupo I: Composto por 90 crianças alunas da primeira série do ensino fundamental do município de Bauru, estado de São

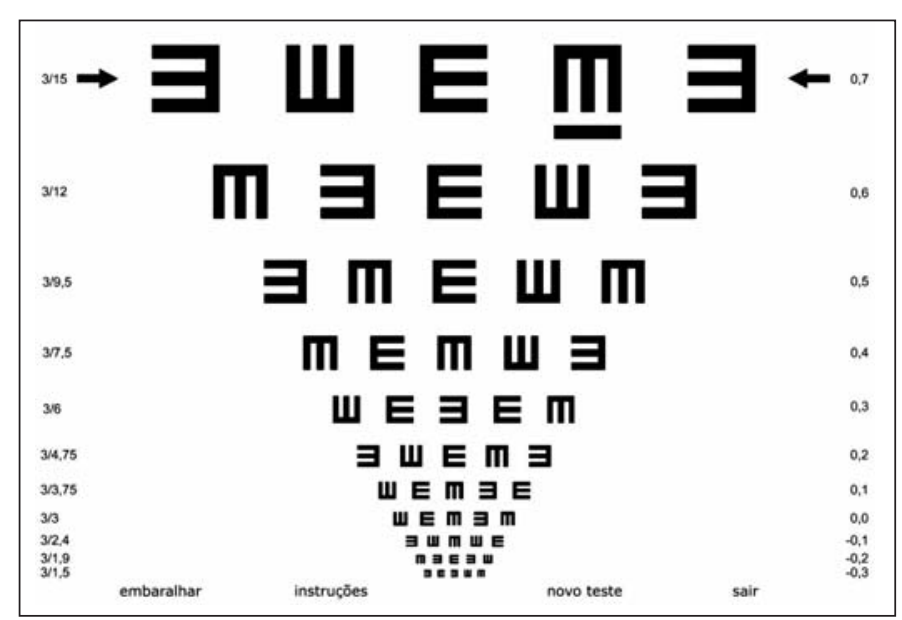

Figura 1 - Ilustração do teste computadorizado 
Paulo, todas com idade de 7 anos, sendo 54 meninas e 36 meninos, após autorização prévia;

Grupo II: Composto por 8 adultos sem alterações oculares, com idade média de 21 anos (variando de 20 a 23 anos);

Grupo III: Composto por 10 pacientes do setor de Estrabismo do Departamento de Oftalmologia da UNIFESP-EPM selecionados ao acaso, com idade variando de 6 a 29 anos (média \pm desvio-padrão de $11 \pm 7,62$ ). As alterações oculares dos sujeitos do Grupo III são descritas na tabela 1 .

Recebemos o consentimento livre, após esclarecimento sobre o trabalho de pesquisa, de todos os adultos voluntários e dos pais das crianças testadas.

Todos os voluntários foram submetidos à avaliação da AV de ambos os olhos através da tabela logarítmica de optotipos E e do programa computadorizado.

A acuidade visual foi determinada separadamente para cada olho, pelo mesmo examinador. A escolha do primeiro método a ser utilizado, bem como do primeiro olho a ser testado, foi definida por sorteio.

Para avaliação através da tabela impressa de optotipos E, os sujeitos foram posicionados sentados a 3 metros de distância da tabela impressa, com um olho ocluído por vez e instruídos a identificar a orientação do optotipo E apresentado.

Para avaliação da AV através do programa computadorizado, os sujeitos foram posicionados sentados a 3 metros de distância do monitor do computador e o método de aplicação do teste foi o mesmo adotado para o exame com a tabela de optotipos E impressa.

Inicialmente, foram apresentadas duas letras de cada fileira, até que um erro na identificação da posição fosse cometi$\mathrm{do}^{(20)}$. Após o primeiro erro, foram apresentados 5 optotipos por fileira, a partir da linha anterior à do erro, e a apresentação continuou, mesmo após a linha de visão 0,0 logMAR para determinação da acuidade máxima do sujeito testado. Os erros

\begin{tabular}{|c|c|c|c|}
\hline & $\begin{array}{l}\text { Idade } \\
\text { (anos) }\end{array}$ & Diagnóstico & Fundo de Olho \\
\hline 1 & 29 & $\begin{array}{l}\text { Endomicrotropia residual, } \\
\text { ambliopia no olho direito }\end{array}$ & Normal nos dois olhos \\
\hline 2 & 9 & $\begin{array}{l}\text { Endotropia motora precoce, } \\
\text { ambliopia no olho direito }\end{array}$ & Normal nos dois olhos \\
\hline 3 & 11 & Endotropia congênita & Normal nos dois olhos \\
\hline 4 & 8 & Endotropia congênita & Normal nos dois olhos \\
\hline 5 & 9 & $\begin{array}{l}\text { Endotropia motora precoce, } \\
\text { ambliopia no olho esquerdo }\end{array}$ & $\begin{array}{l}\text { Tortuosidade de vasos } \\
\text { nos dois olhos }\end{array}$ \\
\hline 6 & 8 & $\begin{array}{l}\text { Monofixação foveal, } \\
\text { ambliopia no olho esquerdo }\end{array}$ & Normal nos dois olhos \\
\hline 7 & 7 & Endotropia congênita & Normal nos dois olhos \\
\hline 8 & 11 & $\begin{array}{l}\text { Exotropia básica, } \\
\text { ambliopia no olho direito }\end{array}$ & $\begin{array}{l}\text { Cicatriz de corio perima- } \\
\text { cular no olho direito; nor- } \\
\text { mal no olho esquerdo }\end{array}$ \\
\hline 9 & 6 & Endotropia motora precoce & Normal nos dois olhos \\
\hline 10 & 23 & $\begin{array}{l}\text { Endotropia motora precoce, } \\
\text { ambliopia no olho esquerdo }\end{array}$ & Normal nos dois olhos \\
\hline
\end{tabular}

e acertos dos 2 olhos de cada sujeito avaliado através dos 2 testes (computadorizado e padrão-ouro) foram marcados em uma ficha de avaliação, que apresenta uma cópia em tamanho reduzido dos testes. A AV foi obtida quando pelo menos 3 letras de uma fileira foram identificadas corretamente, e nenhuma letra da fileira seguinte foi identificada. Para análise do real índice de $\mathrm{AV}$, os dados foram interpolados e registrados em unidade logarítmica.

Consideramos o índice de AV de cada letra identificada corretamente. Se cada fileira possui 5 optotipos e entre fileiras consecutivas a diferença é de 0,1 $\log$ MAR, cada optotipo representa $0,02 \log$ MAR. Se apenas 3 optotipos foram corretamente identificados na fileira 0,0 logMAR, o índice real interpolado da AV dele está entre 0,0 logMAR e 0,1 logMAR correspondendo a $0,06 \log \mathrm{MAR}^{(21)}$.

Acuidade visual 0,0 $\log$ MAR corresponde a acuidade 20/20 (ou 1,0) de Snellen, e 1,0 logMAR corresponde a acuidade 20/200 (ou 0,1 de Snellen). Portanto, quanto menor o índice da AV logarítmica, melhor a discriminação visual do sujeito.

Para análise dos resultados, acuidade visual 20/20 (0,0 $\log$ MAR) foi definida como "normal" e AV menor ou igual a 20/25 (a partir de 0,12 logMAR), como "alterada”.

O programa computadorizado apresenta dez páginas com orientações para a realização do procedimento. Inclue instruções para: ajuste de acordo com o tamanho do monitor utilizado, ajustes do brilho e contraste, centralização da imagem na tela do computador e paginas de teste. Foi desenvolvido para identificar a resposta marcada pelo examinador com o teclado de setas como certa ou errada e automaticamente decidir se a apresentação deve continuar para a fileria de letras seguinte (no caso de resposta correta) ou se deve voltar uma fileira para apresentação de optotipo por optotipo.

\section{RESULTADOS}

Para análise dos resultados, optamos por selecionar a do olho de melhor AV, determinada através da tabela de optotipos (padrão-ouro). Quando os resultados dos olhos direito e esquerdo foram os mesmos, selecionamos o olho de melhor AV determinada pelo programa computadorizado.

As figuras 2, 3 e 4 apresentam os gráficos demonstrativos dos resultados do melhor olho, determinado pelo padrãoouro, dos sujeitos dos grupos I, II e III, respectivamente.

Analisando os 90 olhos direitos e esquerdos dos sujeitos do grupo I, encontramos uma tendência do teste computadorizado em superestimar os valores da $\mathrm{AV}$, quando comparado com a tabela impressa.

Para melhor análise dessa tendência, aplicamos o teste do sinal e notamos a ocorrência de um erro sistemático, $\mathrm{p}=0,040$ $\log$ MAR para o olho direito e $\mathrm{p}=0,009 \log$ MAR para o olho esquerdo, com o teste feito no computador apresentando valores maiores do que o teste feito com a tabela de optotipos impressa. Para entendermos essa diferença (média) realizamos o teste T. O teste t-pareado mostra uma diferença, em média, 


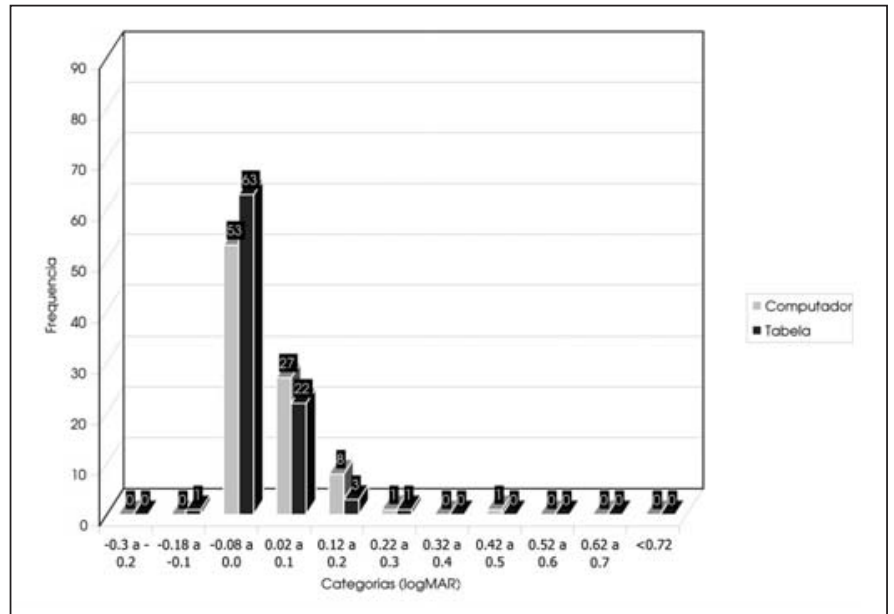

Figura 2 - Gráfico demonstrativo dos resultados do melhor olho, determinado pelo padrão-ouro, dos 90 sujeitos do grupo I

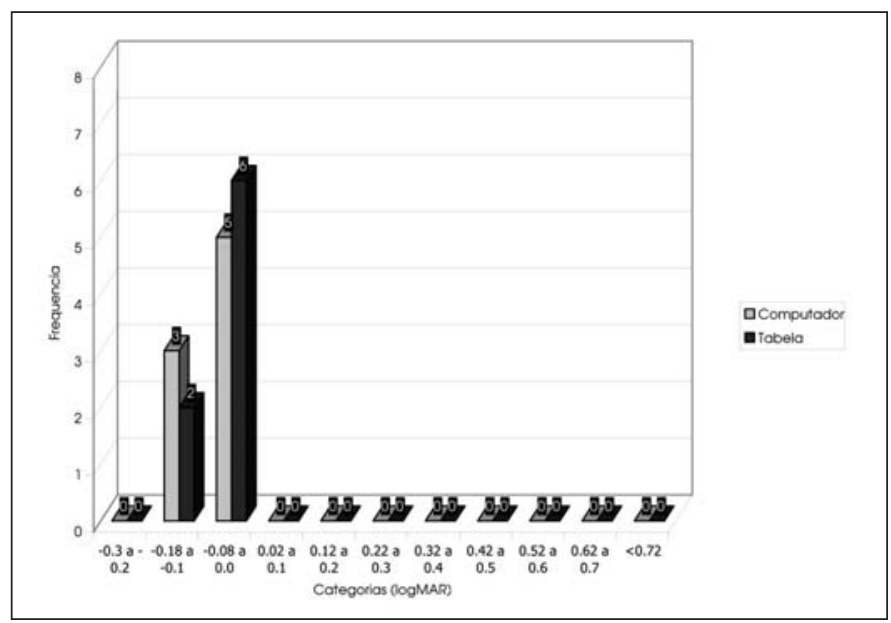

Figura 3 - Gráfico demonstrativo dos resultados do melhor olho, determinado pelo padrão-ouro (tabela de optotipos impressa), dos sujeitos do grupo II

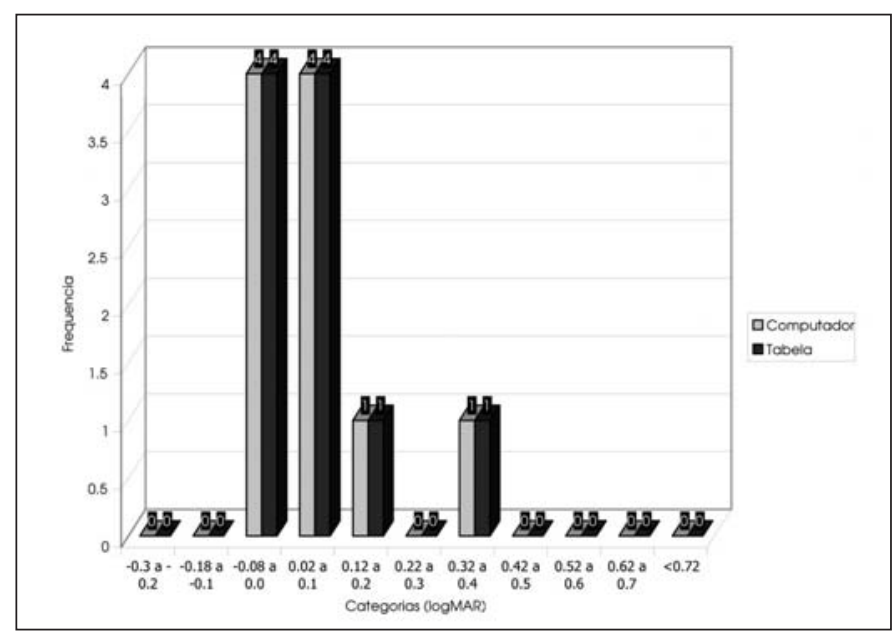

Figura 4 - Gráfico demonstrativo dos resultados do melhor olho, determinado pelo padrão-ouro (tabela de optotipos E impressa), dos sujeitos do grupo III estatisticamente significante entre os testes $(0,011 \log$ MAR para o olho direito e 0,0149 logMAR para o olho esquerdo). Para efeito de análise dos resultados, nós definimos AV normal como sendo a que se encontra dentro do intervalo de - 0,3 $\log$ MAR a $0,1 \log$ MAR e, portanto, a diferença encontrada no teste T é clinicamente não-significante, abrangendo menos do que um optotipo em cada olho (0,02 logMAR).

O coeficiente de correlação intraclasse obtido foi de 0,8211 (IC $[0,7405 ; 0,8784]$ ) para os 90 olhos direitos e de 0,7868 (IC $[0,6935 ; 0,8542]$ ) para os 90 olhos esquerdos dos 90 alunos da primeira série, sendo essas correlações consideradas excelentes, pois $r>0,75^{(22)}$.

Outra análise de concordância entre os dois testes para classificação do melhor olho foi realizada através do coeficiente de kappa. O resultado encontrado no grupo dos 90 alunos da primeira série caracterizou razoável concordância entre a tabela e o método computadorizado para a determinação do melhor olho $(\mathrm{k}=0,332)^{(23)}$.

A concordância entre os dois testes também foi avaliada através do método de Bland Altman, que representa a magnitude da concordância entre os dois métodos (Figuras 5 e 6). Os pontos da figura mostram, para cada paciente, a diferença entre as acuidades dos dois métodos (AV tabela - AV computador) contra a média da AV ([AV tabela + AV computador $] / 2$ ). Não observamos 90 pontos distintos pois, em alguns casos, os pontos estão sobrepostos ${ }^{(24)}$.

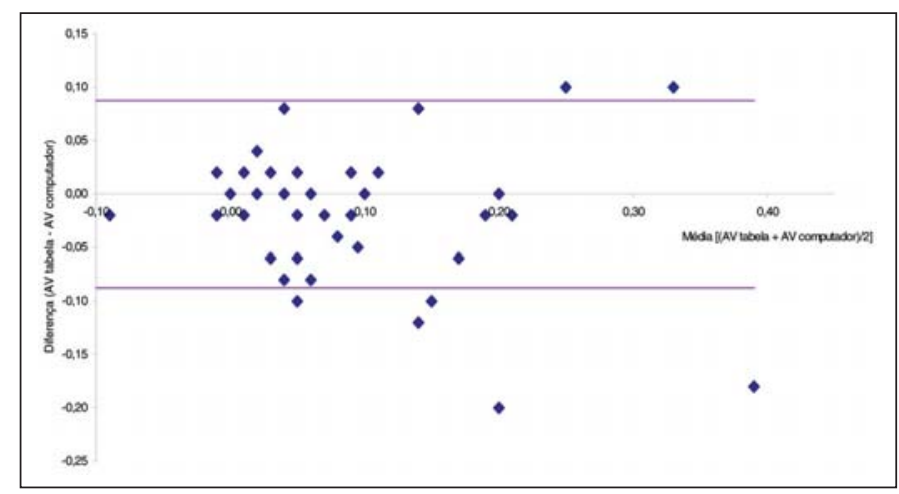

Figura 5 - Análise de Bland Altman para o grupo I (olho direito)

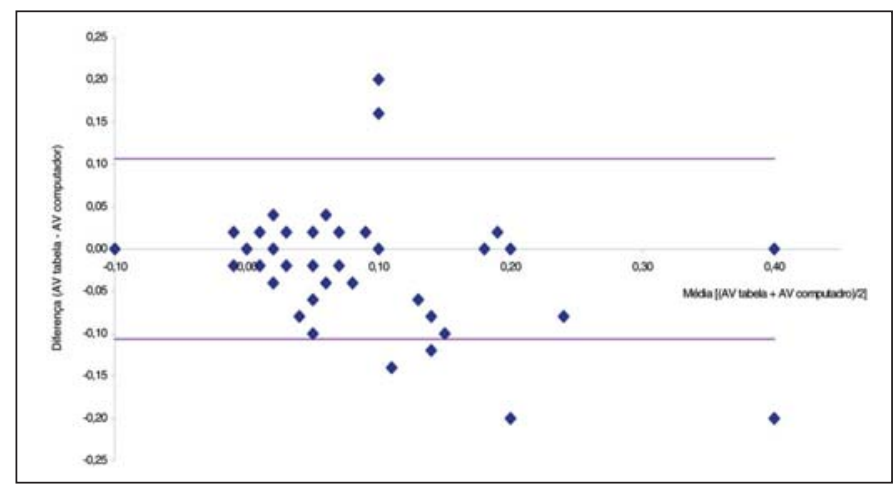

Figura 6 - Análise de Bland Altman para o grupo I (olho esquerdo) 
A mesma tendência do teste computadorizado em superestimar os valores da AV foi encontrada quando analisamos os 10 olhos direitos e os 10 olhos esquerdos dos sujeitos do grupo III, através do computador e da tabela impressa. O teste t-pareado não mostrou diferença estatisticamente significante (em média) entre os testes $(\mathrm{p}=0,496 \log$ MAR para o olho direito e $\mathrm{p}=0,210 \log$ MAR para o olho esquerdo) talvez devido ao tamanho reduzido da amostra que compunha o grupo III.

O coeficiente de correlação intraclasse obtido foi de 0,9934 (IC $[0,9753 ; 0,9983])$ para os 10 olhos direitos e de 0,9851 (IC $[0,9447 ; 0,9962])$ para os 10 olhos esquerdos dos sujeitos do grupo III, sendo essas correlações consideradas excelentes pois $r>0,75^{(22)}$.

Outra análise de concordância entre os dois testes para classificação do melhor olho foi realizada através do coeficiente de kappa. O resultado encontrado no grupo III caracterizou a concordância como quase perfeita entre a tabela de optotipos impressa e o método computadorizado para a determinação do melhor olho $(\mathrm{k}=0,848)^{(23)}$.

A concordância entre os dois testes também foi avaliada através do método de Bland Altman (Figuras 7 e 8) que representou o tamanho da concordância entre os dois métodos. Os pontos da figura mostram, para cada paciente, a diferença entre as acuidades dos dois métodos (AV tabela - AV computador) juntamente com a média da AV ([AV tabela + AV com-

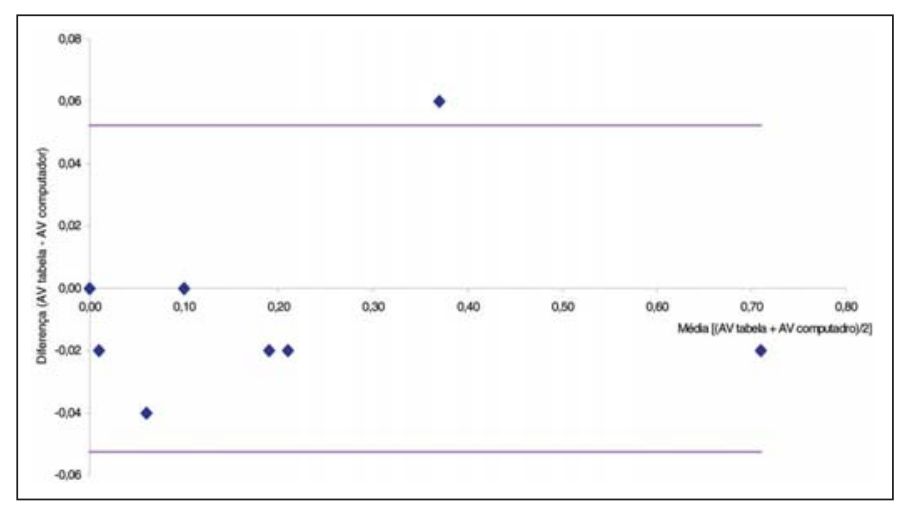

Figura 7 - Análise de Bland Altman para o grupo III (olho direito)

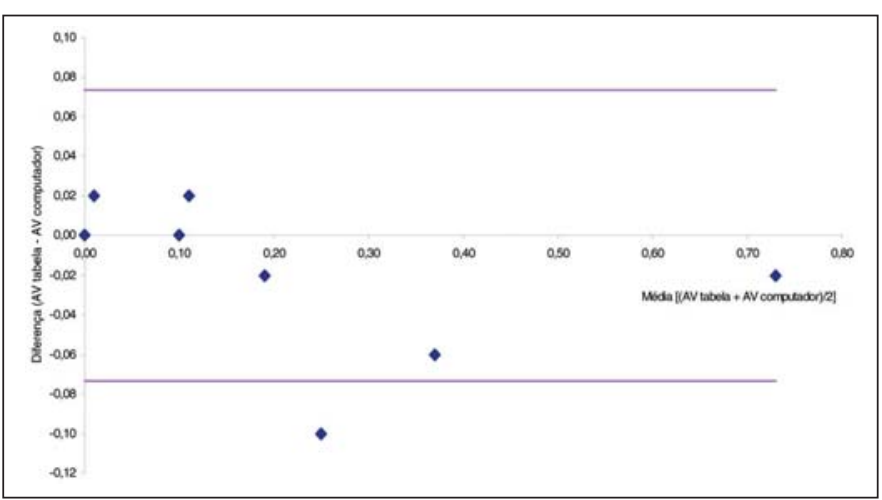

Figura 8 - Análise de Bland Altman para o grupo III (olho esquerdo) putador]/2). Não observamos 10 pontos distintos pois, em alguns casos, os pontos estão sobrepostos ${ }^{(24)}$.

Comparando os resultados do grupo II determinados através da tabela impressa de optotipos e do programa computadorizado (Figura 3), podemos dizer que para a avaliação dos sujeitos desse grupo os dois métodos foram equivalentes e, por isso, não foi possível realizar análise estatística dos resultados dos olhos normais.

Para determinarmos a sensibilidade e a especificidade do teste computadorizado, comparamos os resultados obtidos com o padrão-ouro (tabela impressa). A sensibilidade de um teste indica a probabilidade desse teste identificar corretamente as pessoas que possuem alterações visuais. A especificidade de um teste é a probabilidade do teste identificar corretamente as pessoas que possuem visão normal.

Para a realização de tal análise, mantivemos os resultados de acordo com o olho de melhor AV determinada pelo padrãoouro para comparação.

No grupo I, encontramos 86 olhos com AV normal (até 0,1 $\log$ MAR) e 4 olhos com baixa de AV (de 0,12 logMAR ou mais) determinada através do padrão-ouro (Tabela). Pelo teste computadorizado, foram encontrados 80 olhos com AV normal e 10 olhos com baixa de AV. Comparando-se os resultados do grupo I, o teste computadorizado apresentou sensibilidade de $100 \%$ e especificidade de $93 \%$ (Tabela 2 ).

No grupo II, encontramos 8 olhos com AV normal e nenhum olho com baixa de AV. O teste computadorizado apresentou sensibilidade de $100 \%$ e especificidade de $100 \%$, pois concordou com o padrão-ouro na afirmação de que nenhum olho apresenta baixa de AV e 8 olhos apresentam AV normal (Tabela 3).

No grupo III, encontramos 8 olhos com AV normal e 2 olhos com baixa de AV pelo padrão-ouro, e pela comparação dos resultados, o teste computadorizado apresentou sensibilidade e especificidade de $100 \%$ (Tabela 4).

\begin{tabular}{|c|c|c|c|}
\hline \multirow[b]{2}{*}{ Computador } & \multicolumn{3}{|c|}{ Padrão-ouro } \\
\hline & BAV & NL & Total \\
\hline BAV & 4 & 6 & 10 \\
\hline NL & 0 & 80 & 80 \\
\hline Total & 4 & 86 & 90 \\
\hline
\end{tabular}

\begin{tabular}{|l|ccc|}
\hline $\begin{array}{l}\text { Tabela } \\
\text { método computadorizado para medida da AV dos } 8 \text { olhos do grupo II }\end{array}$ \\
\cline { 2 - 4 } Computador & BAV & Padrão-ouro & \\
BAV & 0 & 0 & Total \\
NL & 0 & 8 & 0 \\
Total & 0 & 8 & 8 \\
BAV= baixa de acuidade visual; $N L=$ acuidade visual normal & 8 \\
\hline
\end{tabular}


Analisando os dados dos 108 olhos estudados, podemos afirmar que a sensibilidade geral do teste computadorizado é de $100 \%$ (identificou corretamente os 6 olhos com baixa de AV) e a especificidade geral é de $94 \%$, pois falhou na classificação de $6 \%$ dos olhos com AV normal (Tabela 5).

\section{DISCUSSÃO}

Apesar da grande evolução tecnológica alcançada pela oftalmologia, ainda não somos capazes de prevenir as alterações visuais detectáveis pelo simples exame da acuidade visual nas crianças.

A base da saúde visual deve ser estruturada e fortalecida nos primeiros anos de vida, enquanto é possível reverter e tratar alterações como ambliopia. Deparamos-nos com uma contradição: grande avanço tecnológico que beneficia o controle e o diagnóstico de doenças importantes, como as degenerações da retina, mas pouca atenção à prevenção, à garantia do desenvolvimento visual normal nos casos de crianças com menos de 7 anos de idade. Por que não unir a tecnologia à prevenção? Essa dúvida impulsionou a proposta de criarmos um programa computadorizado para auxiliar na triagem visual de escolares.

Teoricamente, podemos dizer que a triagem visual deveria acontecer aos 6 meses de idade, para avaliação do estado de fixação e alinhamento ocular e, a partir dos 3 anos de idade, através das técnicas quantitativas de medida da $\mathrm{AV}^{(25)}$. Podemos dizer que o objetivo da realização da triagem visual é identificar, entre indivíduos aparentemente normais, aqueles com risco de desenvolver um defeito visual, na esperança de maximizar as chances de cura quando o tratamento precoce é instituído ${ }^{(5)}$.

Triagem visual de crianças com menos de 6 anos de idade é possível através, por exemplo, do teste E de Snellen mas a

\begin{tabular}{|c|c|c|c|}
\hline \multirow[b]{2}{*}{ Computador } & \multicolumn{3}{|c|}{ Padrão-ouro } \\
\hline & BAV & NL & Total \\
\hline BAV & 2 & 0 & 2 \\
\hline NL & 0 & 8 & 8 \\
\hline Total & 2 & 8 & 10 \\
\hline
\end{tabular}

\begin{tabular}{|lccc|}
\hline \multicolumn{3}{|l|}{$\begin{array}{l}\text { Tabela 5. Concordância entre o padrão-ouro (tabela impressa) e o } \\
\text { método computadorizado para medida da AV dos 108 olhos estudados }\end{array}$} \\
\cline { 2 - 4 } Computador & BAV & Padrão-ouro \\
\hline BAV & 6 & 6 & Total \\
NL & 0 & 96 & 12 \\
Total & 6 & 102 & 96 \\
BAV= baixa de acuidade visual; NL= acuidade visual normal & 108 \\
\hline
\end{tabular}

dificuldade na orientação de lateralidade prejudica a sua aplicação ${ }^{(26)}$. Outro fator que dificulta tal aplicação é a fraca colaboração e desatenção típicas nesta faixa etária ${ }^{(27)}$. Para resolver este problema, os professores e orientadores educacionais poderiam instruir os pais dessas crianças para que procurassem um serviço oftalmológico para adequada avaliação da função visual de seus filhos. Mas a situação sócio-econômica do país não favorece tal procedimento, principalmente nas classes menos favorecidas. As crianças que conseguem garantir uma vaga na escola pública dificilmente terão acesso à consulta oftalmológica através de convênios médicos, planos de saúde ou atendimento particular. As queixas visuais dificilmente aparecem antes da idade escolar e, na ausência de sinais ou sintomas que alertem para um problema visual, o exame oftalmológico não é realizado ${ }^{(16,28)}$.

A participação de outras pessoas da comunidade, além dos oftalmologistas, é necessária para o combate às perdas visuais preveníveis na infância ${ }^{(19)}$.

Durante o primeiro ano do ensino fundamental, a criança inicia seu processo formal de ensino-aprendizagem, considerado um dos momentos mais importantes da socialização e necessita da visão e audição normais (ou com correção adequada) para que esse processo seja facilitado. Crianças com visão e/ou audição deficientes podem ter seu desempenho escolar prejudicado ${ }^{(29)}$. Os distúrbios visuais não corrigidos contribuem para a deficiência no aproveitamento escolar, distúrbios emocionais, psicológicos, prejuízos no desenvolvimento da personalidade, além da ambliopia ${ }^{(16)}$.

A necessidade da detecção precoce das alterações visuais está clara mas é inviável obter avaliação oftalmológica profissional para toda a população. Como alternativa, podemos instituir triagens em estudantes da primeira série do ensino fundamental de escolas públicas e privadas ${ }^{(11)}$. Nesta faixa etária, as dificuldades para avaliação da acuidade visual são menores pois as crianças conseguem entender e participar do teste, mesmo que necessitem de treinamento prévio, além de não dependerem da iniciativa dos pais para procurar avaliação visual. A triagem visual na escola não requer gastos e investimentos e poderia ser incluída como regra em todas as escolas primárias, contribuindo com o diagnóstico precoce de alterações da visão.

Encontramos estudos na literatura nacional que relatam os resultados da realização de triagem visual em escolas primárias no Brasil ${ }^{(17-18,26,30)}$. Geralmente os testes são realizados com o uso das tabelas impressas de Snellen e aplicados por professores treinados, nas escolas, durante o período de aula, e demonstram diferentes índices de alterações visuais entre os escolares. Cano e Silva (1994) referem 6,3\% de distúrbios visuais entre os alunos de primeira série ${ }^{(29)}$; Carvalho e Garrido (1993) relatam 36,5\% de crianças na faixa etária entre 4 e 13 anos com baixa de AV no Amazonas ${ }^{(18)}$; Figueiredo et al., (1993) encontraram 12\% de problemas oftalmológicos entre escolares da primeira e segunda série do ciclo básico da cidade de São Carlos ${ }^{(26)}$. 
Esses índices não foram confirmados durante a avaliação das 90 crianças alunas da primeira série do ensino fundamental de Bauru, interior de São Paulo, o que não desqualifica o método de triagem proposto. $\mathrm{O}$ fato de não termos encontrado índices de alterações visuais entre os escolares examinados pode ser explicado em parte pela existência da Campanha Nacional de Prevenção da Cegueira e Reabilitação Visual "Veja Bem Brasil", implantada em 1998, que visa apresentar a incidência das doenças oculares na infância e promover a saúde ocular encaminhando precocemente as crianças ao tratamento necessário ${ }^{(17)}$.

O sucesso da triagem visual tradicional para o diagnóstico precoce das alterações visuais depende do treinamento do aplicador, evitando os erros sistemáticos como os causados pela apresentação dos optotipos de maneira errada, interpretação incorreta das respostas, mau posicionamento da criança e memorização da seqüência das letras. Depois de compreendida a importância e o procedimento do exame, os professores estariam aptos a aplicá-lo e contribuir para a detecção de escolares com baixa visual.

Além do treinamento, é importante garantir a padronização do procedimento do teste. A distância teste, a qualidade da impressão das tabelas, sua manutenção e os cuidados com o ambiente onde se realiza a avaliação interferem diretamente no resultado da triagem. É um teste subjetivo, com respostas dependentes do entendimento da criança e interpretação do examinador. Alguns autores publicaram um estudo comparativo entre os resultados de medida da AV realizada por um oftalmologista e por uma atendente de enfermagem, para avaliar a influência do examinador no resultado, e encontraram boa correlação entre os resultados obtidos através do exame aplicado por ambos os examinadores ${ }^{(31)}$.

A automatização do procedimento garante as padronizações necessárias, facilita a aplicação e diminue as variações inter e intra-examinadores com relação aos critérios de avaliação.

Quando idealizamos a construção do teste computadorizado, pensávamos em desenvolver um novo método para triagem da visão das crianças na escola, que substituísse ou complementasse o teste tradicional com as tabelas impressas.

Desenvolvemos um teste que possibilita o controle rígido do estímulo apresentado e dificulta a memorização das letras pela alteração automática da seqüência e ordem dos optotipos apresentados. Por ser automático, minimiza o treinamento prévio necessário para aplicação pois interpreta as respostas dadas pela criança examinada durante o teste e permite o registro e arquivo automático dos resultados. A participação do examinador fica dedicada à instrução, posicionamento adequado e monitoração durante o procedimento, que sempre é realizado pelo programa computadorizado.

Além de automático o programa gera optotipos em progressão logarítmica. Apresenta 11 fileiras (de 0,7 logMAR a -0,3 $\log$ MAR) com cinco optotipos "E" cada. O programa apresenta uma linha vermelha sublinhando o optotipo em teste e o aplicador marca a resposta dada pelo aluno utilizando o teclado de setas. Automaticamente, o programa desenvolve o teste e determina a AV do aluno testado. O aplicador não precisa interpretar a resposta. Esse procedimento possibilita o cadastro de cada aluno testado e o envio do registro da visão para um banco de dados, o que permite a análise à distância dos resultados por um oftalmologista responsável, que orientaria cada caso.

Atualmente, todas as escolas públicas municipais e estaduais podem adquirir, se ainda não tiverem, um computador para as mais diversas funções, inclusive triar a visão dos seus alunos.

Durante a validação do programa computadorizado, todos os voluntários informaram preferência pelo teste computadorizado, por ter sido mais atrativo e dinâmico, quando comparado ao procedimento padrão com a tabela impressa.

Consideramos o padrão-ouro (tabela logarítmica impressa de optotipos E) como tendo alta especificidade e sensibilidade, e analisamos estatisticamente os resultados obtidos através dos dois procedimentos. O teste do sinal demonstrou a ocorrência de um erro sistemático quando a medida da acuidade foi realizada pelo método computadorizado nos dois grupos (alunos da primeira série e nos pacientes do setor de estrabismo), superestimando discretamente a acuidade visual. O efeito aprendizagem foi corrigido pela aleatorização da ordem de apresentação dos testes para cada sujeito, determinada previamente pelo examinador como descrito anteriormente.

Genericamente, podemos dizer que o teste computadorizado apresenta alta sensibilidade e especificidade. Analisando os resultados dos 108 olhos estudados, obtidos com o teste computadorizado, e comparando-os com os resultados do Padrão-ouro, encontramos sensibilidade máxima (100\%) para determinar os olhos com baixa de AV e especificidade geral de $94 \%$ para determinar os olhos com visão normal. Podemos aceitar esse resultado e caracterizar o teste computadorizado como válido, mas jamais substituto do padrão-ouro.

O teste computadorizado permite o controle rígido da apresentação do teste e garante a padronização do procedimento, independente do local de aplicação e do examinador. Por obedecer a progressão logarítmica, mantém o grau de dificuldade inalterado, da primeira à última linha do teste ${ }^{(32)}$.

\section{CONCLUSÕES}

No Brasil, com suas dimensões continentais e diferenças econômicas e culturais nos diversos estados, necessitamos do envolvimento de diversos setores da sociedade para que consigamos instituir programas de prevenção da baixa de acuidade visual nos escolares. Nossa contribuição é fornecer esta ferramenta para a triagem visual, um teste computadorizado válido, atraente para as crianças, de simples manuseio, que garante a padronização do procedimento de avaliação da visão, comparável com a tabela logarítmica impressa de optotipos "E". Mais uma ferramenta para a Telemedicina. Uma ferramenta que não substitui o exame oftalmológico tradicional mas que favorece a localização das crianças com visão afetada ou com suspeita de alteração visual. 


\section{AGRADECIMENTOS}

À Profa. Dra. Adriana Berezovsky, Prof. Dr. Daniel Sigulem, Profa. Dra. Mônica Parente Ramos, Profa. Dra. Cristina Muccioli, Profa. Dra. Célia Regina Nakanami, Gisele Grinevicius Garbe, Fabrício Landi de Moraes, Marcelo Hiroshi Ogava, Profa. Maria José de Oliveira Faustine, Profa. Setsuko Katayama Kjaer, Departamento de Oftalmologia e ao Departamento de Informática em Saúde da UNIFESP-EPM.

\section{ABSTRACT}

Purpose: To elaborate and to validate a computerized test for visual acuity screening of school-age children. Methods: We have created a computerized test for visual acuity assessment with optotypes arranged as those of printed logarithmic charts used in ophthalmic clinic. Ninety seven-year-old students, 8 normal adult volunteers and 10 patients from the Strabismus sector of the Federal University of São Paulo were evaluated by the same examiner and submitted to the visual acuity test through printed visual acuity logarithmic tumble "E" chart and the new computerized test at the same time. Written consent was obtained after clarification about the research project. Results: Statistical analysis showed excellent correlation between the two methods $(r>0.75)$ besides the slight trend of the computerized test to overestimate visual acuity when compared with the gold standard. Sensitivity of the computerized test was $100 \%$ (correctly identified 6 eyes with poor visual acuity) and specificity was $94 \%$. Conclusions: The computerized test can be used as a new clinical tool for visual acuity screening of school-age children and it is fast, easy to perform and inexpensive, besides being more attractive for children. The method releases the examiner from the interpretation of the subject's answers and ensures the procedure's standardization even when more than one examiner performs the test. To better understand the effectiveness of this method for visual screening, one option would be to introduce it in elementary schools, after training the teachers to perform this test.

Keywords: Visual acuity; Vision screening; Software validation; Vision; Students

\section{REFERÊNCIAS}

1. Teller DY, Boothe R. Development of vision in infant primates. Trans Ophthalmol Soc UK. 1979;99(3):333-7.

2. Marg E, Freeman DN, Peltzman P, Goldstein PJ. Visual acuity development in human infants: evoked potential measurements. Invest Ophthalmol Vis Sci. 1976;15:150-3

3. Salomão SR, Ventura DF. Large sample population age norms for visual acuity obtained with vistech-teller acuity cards. Invest Ophthalmol Vis Sci. 1995;36(3):657-70.
4. Catford GV, Oliver A. Development of visual acuity. Arch Dis Child. 1973; 48(1):47-50.

5. Sjöstrand J, Abrahamsson M. Risk factors in amblyopia. Eye. 1990;4(Pt6): 787-93.

6. Simon JW, Kaw P. Commonly missed diagnosis in the childhood eye examination. Am Fam Physician. 2001;64(4):623-8.

7. Ikeda $\mathrm{H}$, Wright MJ. Is amblyopia due to inappropriate stimulation of the "sustained" pathway during development? Br J Ophthalmol. 1974;58(3):165-75.

8. Duane TD. Treatment of the sensorial adaptations and amblyopia. In: Duane TD, editor. Clinical Ophthalmology. Hagerstown, MD: Harper and Row; 1989. p.1-14.

9. Campos E. Amblyopia. Surv Ophthalmol. 1995;40(1):23-39.

10. Rosser DA, Laidlaw DAH, Murdoch IE. The development of a "reduced $\log$ MAR" visual acuity chart for use in routine clinical practice. Br J Ophthalmol. 2001;85(4):432-6.

11. Ehrlich MI, Reinecke RD, Simons K. Preschool vision screening for amblyopia and strabismus. Programs, methods, guidelines, 1983. Surv Ophthalmol. 1983;28(3):145-63.

12. Raymond JE, Shapiro KL. Usefulness of computerized visual acuity testing in a pediatric ophthalmology clinic. Can J Ophthalmol. 1988;23(5):213-15.

13. Moke PS, Turpin AH, Beck RW, Holmes JM, Repka MX, Birch EE, et al. Computerized method of visual acuity testing: adaptation of the amblyopia treatment study visual acuity testing protocol. Am J Ophthalmol. 2001;132 (6): $903-9$

14. Hammond RS, Schmidt PD. A random dot E stereogram for the vision screening of children. Arch Ophthalmol. 1986;104(1):54-60.

15. Flocks M. Visual screening via television. Surv Ophthalmol. 1983;28(3):184-7.

16. Trindade JC. Testes de triagem para avaliação das acuidades visual e auditiva em escolares. Pediatr Mod. 1991;26(5):336-8.

17. Silveira RC, Villano DM, Interlandi R, Rehder JRCL. "Veja Bem Brasil": Oftalmologia Preventiva. Arq Med ABC. 1999;22(1/2):47-9.

18. Carvalho R, Garrido C. Avaliação oftalmológica primária em escolares no Estado do Amazonas, Brasil - I: Cabury/Parintins, Bairro União/Manaus. Rev Bras Oftalmol. 1993;52(5):41-3.

19. Schimiti RB, Costa VP, Gregui MJF, Kara-José N, Temporini ER. Prevalence of refractive errors and ocular disorders in preschool and schoolchildren of Ibiporã - PR, Brazil (1989 to 1996). Arq Bras Oftalmol. 2001;64(5):379-84.

20. Sakuna JT. Medidas de acuidade visual com tabelas logarítmicas: comparação entre métodos psicofísicos. Rev Bras Oftalmol. 1991;50(3):55-64.

21. Treutwein B. Adaptive psychophysical procedures. Vision Res. 1995;35(17): 2503-22.

22. Fleiss JL. The design and analysis of clinical experiments. New York: John Wiley \& Sons.; 1986.

23. Everitt BS. The analysis of contingency tables. London: Chapman \& Hall; 1986.

24. Bland JM, Altman DG. Statistical methods for assessing agreement between two methods of clinical measurement. Lancet. 1986;1(8476):307-10. Comment in: Lancet. 2004;363(9403):164; author reply 164-5.

25 . Eye examination and vision screening in infants, children, and young adults by pediatricians. American Academy of Pediatrics Committee on Practice and Ambulatory Medicine, Section on Ophthalmology. Pediatrics. 1996;98(1):153-7.

26. Figueiredo RM, Santos EC, Jesus IAA, Castilho RM, Santos EV. Proposição de procedimento de detecção sistemática de perturbações oftalmológicas em escolares. Rev Saúde Publica. 1993;27(3):204-9.

27. Veitzman S, Goia DM. Respostas obtidas pela compreensão de dois testes de visão: Snellen e LH, aplicados em crianças pré-escolares. Arq Bras Oftalmol. 1988;51(3):121-2.

28. Briscoe D, Lifshitz T, Grotman M, Kushelevsky A, Vardi H, Weizman S, et al. A new computer program for mass screening of visual defects in preschool children. Br J Ophthalmol. 1998;82(4):415-8.

29. Cano MAT, Silva GB. Detecção de problemas visuais e auditivos de escolares em Ribeirão Preto: Estudo comparativo por nível sócio-econômico. Rev Lat Am Enferm. 1994;2(1):57-68.

30. Siqueira GB, Siqueira MCRG. Projeto de oftalmologia sanitária escolar no município de São Romão - Minas Gerais. Rev Bras Oftalmol. 1994;53(5):71-4.

31. Palazzo Neto G, Lauretti Filho A, Romão E. Estudo comparativo entre as medidas de acuidade visual feitas por um oftalmologista e uma atendente de enfermagem. Rev Bras Oftalmol. 1992;51(5):45-9.

32. Sprague JB, Stock LA, Connett J, Bromberg J. Study of chart designs and optotypes for preschool vision screening - I. Comparability of chart designs. J Pediatr Ophthalmol Strabismus. 1989;26(4):189-97. 